\title{
Observability Estimate for the Fractional Order Parabolic Equations on Measurable Sets
}

\author{
Guojie Zheng ${ }^{1,2}$ and M. Montaz $\mathrm{Ali}^{2,3}$ \\ ${ }^{1}$ College of Mathematics and Information Science, Henan Normal University, Xinxiang 453007, China \\ ${ }^{2}$ School of Computational and Applied Mathematics, University of the Witwatersrand (Wits), Johannesburg 2050, South Africa \\ ${ }^{3}$ TCSE, Faculty of Engineering and Built Environment, University of the Witwatersrand (Wits), Johannesburg 2050, South Africa
}

Correspondence should be addressed to Guojie Zheng; guojiezheng@yeah.net

Received 10 January 2014; Accepted 3 March 2014; Published 27 March 2014

Academic Editor: Sheng-Jie Li

Copyright (c) 2014 G. Zheng and M. M. Ali. This is an open access article distributed under the Creative Commons Attribution License, which permits unrestricted use, distribution, and reproduction in any medium, provided the original work is properly cited.

We establish an observability estimate for the fractional order parabolic equations evolved in a bounded domain $\Omega$ of $\mathbb{R}^{n}$. The observation region is $F \times \omega$, where $\omega$ and $F$ are measurable subsets of $\Omega$ and $(0, T)$, respectively, with positive measure. This inequality is equivalent to the null controllable property for a linear controlled fractional order parabolic equation. The building of this estimate is based on the Lebeau-Robbiano strategy and a delicate result in measure theory provided in Phung and Wang (2013).

\section{Introduction}

Let $\Omega$ be a bounded domain in $\mathbb{R}^{n}, n \geq 1$, with real analytic boundary. Let $\omega \subset \Omega$ be a Lebesgue measurable subset with positive measure, and denote the characteristic function of $\omega$ by $\chi_{\omega}$. Let $T>0$. Let $F \subset(0, T)$ be a Lebesgue measurable subset with positive measure, and denote the characteristic function of $F$ by $\chi_{F}$. Now, we define an unbounded operator $A$ in $L^{2}(\Omega)$ as follows:

$$
\begin{aligned}
& D(A)=H^{2}(\Omega) \cap H_{0}^{1}(\Omega), \\
& A v=-\Delta v, \quad \text { for any } v \in D(A) .
\end{aligned}
$$

Let $\left\{\lambda_{i}\right\}_{i=1}^{\infty}, 0<\lambda_{1}<\lambda_{2} \leq \cdots$, be the eigenvalues of $A=-\Delta$, and let $\left\{e_{i}\right\}_{i=1}^{\infty}$ be the corresponding eigenfunctions satisfied that $\left\|e_{i}(x)\right\|_{L^{2}(\Omega)}=1, i=1,2,3, \ldots$, which constitutes an orthonormal basis of $L^{2}(\Omega)$. It is well known that we can define a class of operator $A^{\alpha}(\alpha \geq 0)$ in $L^{2}(\Omega)$ as follows:

$$
\begin{aligned}
& D\left(A^{\alpha}\right)=\left\{\left.v \in L^{2}(\Omega)\left|v=\sum_{i=1}^{\infty} v_{i} e_{i}, \sum_{i=1}^{\infty} \lambda_{i}^{2 \alpha}\right| v_{i}\right|^{2}<\infty\right\}, \\
& A^{\alpha} v=\sum_{i=1}^{\infty} \lambda_{i}^{\alpha} v_{i} e_{i}, \quad \text { where } v=\sum_{i=1}^{\infty} v_{i} e_{i} .
\end{aligned}
$$

Moreover, the operator $A^{\alpha}$ is a self-adjoint operator and $-A^{\alpha}$ is an infinitesimal generator of a strong continuous semigroup $\left\{S_{\alpha}(t)\right\}_{t \geq 0}$. Now, we consider the following linear controlled fractional order parabolic equation:

$$
\begin{aligned}
\partial_{t} y(x, t)+A^{\alpha} y(x, t) & =B u(x, t), \quad(x, t) \in \Omega \times(0, T], \\
y(x, 0) & =y_{0}(x), \quad x \in \Omega,
\end{aligned}
$$

where $\alpha>1 / 2, B$ is a linear bounded operator in $L^{2}(\Omega)$ defined by $B u=\chi_{F}(t) \cdot \chi_{\omega}(x) u, y_{0} \in L^{2}(\Omega)$, and $u(\cdot, t)$ is a control function taken from the space $L^{2}\left(0, T ; L^{2}(\Omega)\right.$ ). We denote $y\left(\cdot ; y_{0}, u\right)$ to be the unique solution of (3) corresponding to the control $u$ and the initial value $y_{0}$. We denote $\|\cdot\|$ and $\langle\cdot, \cdot\rangle$ to be the usual norm and the inner product in $L^{2}(\Omega)$, respectively.

In recent years, extensive research has been devoted to the study of differential equations with fractional orders due to their importance for applications in various branches of applied sciences and engineering. Many important phenomena in signal processing, electromagnetics, crowded systems, and fluid mechanics are well described by fractional differential equation (see [1]). In this paper, we always discuss the fractional Laplacian. The fractional Laplacian $-A^{\alpha}$, with 
$\alpha \in(0,1]$, generates the rotationally invariant $2 \alpha$ stable Lévy process. For $\alpha=1$, this process is the normal Brownian motion $B_{t}$ on $\mathbb{R}^{n}$ (see [2]).

Now, we will focus on the issue of what the controllable property is for the controlled system (3). System (3) is said to be null controllable in time $T$ if for any $y_{0} \in L^{2}(\Omega)$, there exists a control function $u \in L^{2}\left(0, T ; L^{2}(\Omega)\right)$, such that the solutions of (3) matches

$$
y\left(T ; y_{0}, u\right)=0
$$

The problem of null controllability of parabolic equations has also been the object of numerous studies. Extensive related references can be found in [3-7] and the rich works cited therein. Especially, we refer to [5] for a null controllability result for the parabolic equations which plays a crucial role in establishing the main result in our paper. In the above works, the control region $\omega$ is always assumed to contain an open ball. The reason is that the main technique used in the argument, Carleman inequality, is required to construct weight functions. The construction of such functions seems to be not possible, when $\omega$ do not contain a ball. Recently, the null controllability for the parabolic equations with $\omega$ that is a measurable subset of positive measure has been established in [8], where an inequality involving measurable sets for a class of real analytic functions was set up in a skillful way. On the other hand, the classical null controllability for some fractional order parabolic equation was studied in $[9,10]$. In particular, in [9] the authors proved that one-dimension problem is not controllable from the boundary for $\alpha \epsilon$ $(0,1 / 2]$.

By the classical duality argument [11], the controllable properties can be transformed into observability problems on the adjoint system. The adjoint system for (3) may be described as follows:

$$
\begin{gathered}
\partial_{t} \varphi(x, t)=A^{\alpha} \varphi(x, t) \quad(x, t) \in \Omega \times[0, T), \\
\varphi(x, T)=\varphi_{0}(x), \quad x \in \Omega .
\end{gathered}
$$

Thus, the exact null controllability property is equivalent to the existence of a constant $C=C(T)>0$ such that the following inequality holds for every solution of (5):

$$
\|\varphi(x, 0)\|_{L^{2}(\Omega)}^{2} \leq C(T) \int_{F} \int_{\omega}|\varphi|^{2} d x d t .
$$

Inequality (6) is called observability inequality, and the best constant $C(T)$ in inequality (6) will be referred as the observability constant. In this work, we discuss the internal observability estimate for the adjoint system (5) when $\omega$ and $F$ are measurable subsets of $\Omega$ and $(0, T)$, respectively, with positive measure. To the best of our knowledge, this observability estimate has not been studied in the past publications.

The main result of the paper is presented as follows.

Theorem 1. Suppose that $\Omega \subset \mathbb{R}^{n}, n \geq 1$, is a bounded domain with a real analytic boundary and $\omega \subset \Omega$ is a Lebesgue measurable set with positive measure. Let $T>0$, and let $F \subset(0, T)$ be a Lebesgue measurable set with positive measure.
Let $\alpha>1 / 2$. Then, there exists a constant $C=C(\Omega, T, \omega, F, \alpha)$ such that, for any data $\varphi_{0} \in L^{2}(\Omega)$, the solution of (5) satisfied

$$
\|\varphi(x, 0)\|_{L^{2}(\Omega)}^{2} \leq C(\Omega, T, \omega, F, \alpha) \int_{F} \int_{\omega}|\varphi(x, t)|^{2} d x d t .
$$

Observability inequality (7) in Theorem 1 allows for estimating the total energy of the solutions of (5) at time 0 in terms of the partial energy localized in the observation region $F \times \omega$, where $\omega$ and $F$ are measurable subsets of $\Omega$ and $(0, T)$, respectively, with positive measure. This inequality is equivalent to the null controllability property for the controlled system (3).

We proceed as follows. In Section 2, we give some preliminary results. Section 3 is devoted to the proof of Theorem 1.

\section{Preliminary Results}

In this section, we will introduce some notions and preliminary results. Based on classical semigroup theory, we see that the operator $-A^{\alpha}$ is the generator of a semigroup of contraction in $L^{2}(\Omega)$, which we denote by $S_{\alpha}(t), \alpha>1 / 2$. Indeed, the semigroup can be written as follows:

$$
S_{\alpha}(t) \psi=\sum_{i=1}^{\infty} e^{-\lambda_{i}^{\alpha} t}\left\langle\psi, e_{i}\right\rangle_{e_{i}}, \quad \text { for } \psi \in L^{2}(\Omega) .
$$

From this, it follows that

$$
\left\|S_{\alpha}(t) \psi\right\|_{L^{2}(\Omega)} \leq e^{-\lambda_{1}^{\alpha} t}\|\psi\|_{L^{2}(\Omega)},
$$

for any $\alpha>1 / 2$ (see [12]).

Throughout the rest of the paper, the following notation will be used. For each measurable set $A \subseteq \mathbb{R},|A|$ stands for its Lebesgue measure in $\mathbb{R}$. The following lemma is quoted from [13].

Lemma 2. Let $E \subseteq[0, T]$ be a measurable set with a positive measure, and let $l$ be a density point for $E$. Then for each $z>$ 1 , there exists a $l_{1} \in(l, T)$ such that the sequence $\left\{l_{i}\right\}_{i=1}^{\infty}$, given by

$$
l_{i+1}=l+\frac{1}{z^{i}}\left(l_{1}-l\right),
$$

satisfies

$$
\left|E \cap\left(l_{i+1}, l_{i}\right)\right| \geq \frac{1}{2}\left(l_{i}-l_{i+1}\right)
$$

Next, we recall the following results, which play a key role in this paper.

Lemma 3. Let $\Omega$ be a bounded domain in $R^{n}, n \geq 1$. Suppose that $\Omega$ have real analytic boundary. Then, for each subset $\omega \subset \Omega$ with positive measure, there exist two positive constants $C_{1}>1$ and $C_{2}>0$, which only depend on $\Omega, \omega$, such that

$$
\sum_{\lambda_{i} \leq r}\left|a_{i}\right|^{2} \leq C_{1} e^{C_{2} \sqrt{r}} \int_{\omega}\left|\sum_{\lambda_{i} \leq r} a_{i} e_{i}(x)\right|^{2} d x
$$


for each finite $r>0$ and each choice of the coefficients $\left\{a_{i}\right\}_{\lambda_{i} \leq r}$ with $a_{i} \in \mathbb{R}$.

This conclusion can be found in the literature [8].

Next, for each $r>0$, we define $X_{r}=\operatorname{span}\left\{e_{i}(x)\right\}_{\lambda_{i} \leq r}$ and $X_{r}^{\perp}=\operatorname{span}\left\{e_{i}(x)\right\}_{\lambda_{i}>r}$. Indeed, for each $r>0, L^{2}(\Omega)=X_{r} \oplus$ $X_{r}^{\perp}$.

Lemma 4. For any $\xi_{0} \in X_{r}^{\perp}$, it always holds that

$$
\left\|S_{\alpha}(t) \xi_{0}\right\|_{L^{2}(\Omega)}<e^{-r^{\alpha} t}\left\|\xi_{0}\right\|_{L^{2}(\Omega)} .
$$

This lemma can be easily obtained by (8) and (9).

\section{The Proof of Main Result}

Proof. Let $E=\{t \mid t=T-s$, where $s \in F\}$. Then, $|E|=|F|>$ 0 . Let $l$ be a density point for $E$. By Lemma 2, for $z>1$, there exists a $l_{1} \in(l, T)$ and a sequence $\left\{l_{i}\right\}_{i=1}^{\infty}$ satisfied (10) and (11).

We now define a sequence subset of $(0, T)$ as follows:

$$
E_{i}=\left\{t-\frac{l_{i}-l_{i+1}}{4} \mid t \in E \cap\left(l_{i+1}+\frac{l_{i}-l_{i+1}}{4}, l_{i}\right)\right\} .
$$

In fact, $E_{i}$ is a subset of $\left(l_{i+1}, l_{i+1}+(3 / 4)\left(l_{i}-l_{i+1}\right)\right)$, and

$$
\begin{aligned}
\left|E_{i}\right| & =\left|E \cap\left(l_{i+1}+\frac{l_{i}-l_{i+1}}{4}, l_{i}\right)\right| \\
& =\left|E \cap\left\{\left(l_{i+1}, l_{i}\right) \backslash\left(l_{i+1}, l_{i+1}+\frac{l_{i}-l_{i+1}}{4}\right)\right\}\right| .
\end{aligned}
$$

By (11), it follow that

$$
\left|E_{i}\right| \geq \frac{l_{i}-l_{i+1}}{4} .
$$

Let $i_{0}$ be the first natural number satisfying $i_{0}>1 /(2 \alpha-1)$; namely, $i_{0}=[1 /(2 \alpha-1)]+1$. Let $b>1$ be a positive number satisfying

$$
\begin{aligned}
& \left(\frac{b^{\alpha}}{z}\right)^{i}\left(1-\frac{1}{z}\right)\left(l_{1}-l\right)>4 C_{2} b^{(i+1) / 2}+4 \ln \left(8 C_{1} z\right) \text {, } \\
& \text { for } i=i_{0}, i_{0}+1, i_{0}+2, \ldots \text {. }
\end{aligned}
$$

Taking $r_{i}=b^{i}$, by (8), it follows that, for any $\xi \in X_{r_{i}}$,

$$
\begin{aligned}
& \int_{l_{i+1}}^{l_{i+1}+(3 / 4)\left(l_{i}-l_{i+1}\right)} \chi_{E_{i}}(t)\left\|S_{\alpha}\left(l_{i+1}+\frac{3}{4}\left(l_{i}-l_{i+1}\right)\right) \xi\right\|_{L^{2}(\Omega)} d t \\
& \leq \int_{l_{i+1}}^{l_{i+1}+(3 / 4)\left(l_{i}-l_{i+1}\right)} \chi_{E_{i}}(t)\left\|S_{\alpha}(t) \xi\right\|_{L^{2}(\Omega)} d t .
\end{aligned}
$$

Combining with (16) and (12), this shows that

$$
\begin{aligned}
& \frac{l_{i}-l_{i+1}}{4}\left\|S_{\alpha}\left(l_{i+1}+\frac{3}{4}\left(l_{i}-l_{i+1}\right)\right) \xi\right\|_{L^{2}(\Omega)} \\
& \leq\left|E_{i}\right| \cdot\left\|S_{\alpha}\left(l_{i+1}+\frac{3}{4}\left(l_{i}-l_{i+1}\right)\right) \xi\right\|_{L^{2}(\Omega)}
\end{aligned}
$$

$$
\begin{aligned}
& \leq \int_{l_{i+1}}^{l_{i+1}+(3 / 4)\left(l_{i}-l_{i+1}\right)} \chi_{E_{i}}(t)\left\|S_{\alpha}(t) \xi\right\|_{L^{2}(\Omega)} d t \\
& \leq C_{1} e^{C_{2} \sqrt{r_{i}}} \int_{l_{i+1}}^{l_{i+1}+(3 / 4)\left(l_{i}-l_{i+1}\right)} \chi_{E_{i}}(t)\left\|S_{\alpha}(t) \xi\right\|_{L^{2}(\omega)} d t .
\end{aligned}
$$

For each $\phi \in L^{2}(\Omega)$, we can write $\phi=\phi_{1}+\phi_{2}$, where $\phi_{1} \epsilon$ $X_{r_{i}}$ and $\phi_{2} \in X_{r_{i}}^{\perp}$. Taking $\xi=S_{\alpha}\left(\left(l_{i}-l_{i+1}\right) / 4\right) \phi_{1}$ in (19), it follows that

$$
\begin{aligned}
& \frac{l_{i}-l_{i+1}}{4}\left\|S_{\alpha}\left(l_{i}\right) \phi_{1}\right\|_{L^{2}(\Omega)} \\
& \leq \frac{l_{i}-l_{i+1}}{4}\left\|S_{\alpha}\left(l_{i+1}+\frac{3}{4}\left(l_{i}-l_{i+1}\right)\right) \circ S_{\alpha}\left(\frac{l_{i}-l_{i+1}}{4}\right) \phi_{1}\right\|_{L^{2}(\Omega)} \\
& \leq C_{1} e^{C_{2} \sqrt{r_{i}}} \int_{l_{i+1}}^{l_{i+1}+(3 / 4)\left(l_{i}-l_{i+1}\right)} \chi_{E_{i}}(t) \\
& \quad \times\left\|S_{\alpha}\left(t+\frac{l_{i}-l_{i+1}}{4}\right) \phi_{1}\right\|_{L^{2}(\omega)} d t \\
& \leq C_{1} e^{C_{2} \sqrt{r_{i}}} \int_{l_{i+1}+\left(\left(l_{i}-l_{i+1}\right) / 4\right)}^{l_{i}} \chi_{E_{i}}\left(t-\frac{l_{i}-l_{i+1}}{4}\right) \\
& \times\left\|S_{\alpha}(t) \phi_{1}\right\|_{L^{2}(\omega)} d t .
\end{aligned}
$$

By the definition of $E_{i}$, it is easily to see that

$$
\chi_{E_{i}}\left(t-\frac{l_{i}-l_{i+1}}{4}\right)=\chi_{E}(t)
$$

$$
\text { for any } t \in\left(l_{i+1}+\frac{l_{i}-l_{i+1}}{4}, l_{i}\right) \text {. }
$$

This, together with (20), deduces

$$
\begin{aligned}
& \frac{l_{i}-l_{i+1}}{4}\left\|S_{\alpha}\left(l_{i}\right) \phi_{1}\right\|_{L^{2}(\Omega)} \\
& \leq C_{1} e^{C_{2} \sqrt{r_{i}}} \int_{l_{i+1}+\left(\left(l_{i}-l_{i+1}\right) / 4\right)}^{l_{i}} \chi_{E}(t) \\
& \quad \times\left(\left\|S_{\alpha}(t) \phi\right\|_{L^{2}(\omega)}+\left\|S_{\alpha}(t) \phi_{2}\right\|_{L^{2}(\Omega)}\right) d t \\
& \leq C_{1} e^{C_{2} \sqrt{r_{i}}} \int_{l_{i+1}+\left(\left(l_{i}-l_{i+1}\right) / 4\right)}^{l_{i}} \chi_{E}(t)\left\|S_{\alpha}(t) \phi\right\|_{L^{2}(\omega)} d t \\
& \quad+C_{1} e^{C_{2} \sqrt{r_{i}}}\left(l_{i}-l_{i+1}\right)\left\|S_{\alpha}\left(l_{i+1}+\frac{l_{i}-l_{i+1}}{4}\right) \phi_{2}\right\|_{L^{2}(\Omega)} d t .
\end{aligned}
$$

The last step is based on the energy decay property of $S_{\alpha}(t)$. Along with Lemma 4, we derive that

$$
\begin{aligned}
& \frac{l_{i}-l_{i+1}}{4}\left\|S_{\alpha}\left(l_{i}\right) \phi_{1}\right\|_{L^{2}(\Omega)} \\
& \leq C_{1} e^{C_{2} \sqrt{r_{i}}} \int_{l_{i+1}+\left(\left(l_{i}-l_{i+1}\right) / 4\right)}^{l_{i}} \chi_{E}(t)\left\|S_{\alpha}(t) \phi\right\|_{L^{2}(\omega)} d t \\
& \quad+C_{1} e^{C_{2} \sqrt{r_{i}}}\left(l_{i}-l_{i+1}\right) e^{-r_{i}^{\alpha}\left(\left(l_{i}-l_{i+1}\right) / 4\right)}\left\|S_{\alpha}\left(l_{i+1}\right) \phi_{2}\right\|_{L^{2}(\Omega)}
\end{aligned}
$$




$$
\begin{aligned}
\leq & C_{1} e^{C_{2} \sqrt{r_{i}}} \int_{l_{i+1}}^{l_{i}} \chi_{E}(t)\left\|S_{\alpha}(t) \phi\right\|_{L^{2}(\omega)} d t \\
& +C_{1} e^{C_{2} \sqrt{r_{i}}}\left(l_{i}-l_{i+1}\right) e^{-r_{i}^{\alpha}\left(\left(l_{i}-l_{i+1}\right) / 4\right)}\left\|S_{\alpha}\left(l_{i+1}\right) \phi_{2}\right\|_{L^{2}(\Omega)}
\end{aligned}
$$

Therefore,

$$
\begin{aligned}
& \frac{l_{i}-l_{i+1}}{4}\left\|S_{\alpha}\left(l_{i}\right) \phi\right\|_{L^{2}(\Omega)} \leq C_{1} e^{C_{2} \sqrt{r_{i}}} \int_{l_{i+1}}^{l_{i}} \chi_{E}(t)\left\|S_{\alpha}(t) \phi\right\|_{L^{2}(\omega)} d t \\
& +C_{1} e^{C_{2} \sqrt{r_{i}}}\left(l_{i}-l_{i+1}\right) e^{-r_{i}^{\alpha}\left(\left(l_{i}-l_{i+1}\right) / 4\right)}\left\|S_{\alpha}\left(l_{i+1}\right) \phi_{2}\right\|_{L^{2}(\Omega)} \\
& \quad+\frac{l_{i}-l_{i+1}}{4}\left\|S_{\alpha}\left(l_{i}\right) \phi_{2}\right\|_{L^{2}(\Omega)} \\
& \leq C_{1} e^{C_{2} \sqrt{r_{i}}} \int_{l_{i+1}}^{l_{i}} \chi_{E}(t)\left\|S_{\alpha}(t) \phi\right\|_{L^{2}(\omega)} d t \\
& \quad+C_{1} e^{C_{2} \sqrt{r_{i}}}\left(l_{i}-l_{i+1}\right) e^{-r_{i}^{\alpha}\left(\left(l_{i}-l_{i+1}\right) / 4\right)}\left\|S_{\alpha}\left(l_{i+1}\right) \phi_{2}\right\|_{L^{2}(\Omega)} \\
& +\frac{l_{i}-l_{i+1}}{4} e^{-r_{i}^{\alpha}\left(l_{i}-l_{i+1}\right)}\left\|S_{\alpha}\left(l_{i+1}\right) \phi_{2}\right\|_{L^{2}(\Omega)} .
\end{aligned}
$$

Thus,

$$
\begin{aligned}
& \frac{l_{i}-l_{i+1}}{4}\left\|S_{\alpha}\left(l_{i}\right) \phi\right\|_{L^{2}(\Omega)} \\
& \quad \leq C_{1} e^{C_{2} \sqrt{r_{i}}} \int_{l_{i+1}}^{l_{i}} \chi_{E}(t)\left\|S_{\alpha}(t) \phi\right\|_{L^{2}(\omega)} d t \\
& \quad+\left(l_{i}-l_{i+1}\right) e^{-r_{i}^{\alpha}\left(\left(l_{i}-l_{i+1}\right) / 4\right)}\left(C_{1} e^{C_{2} \sqrt{r_{i}}}+1\right)\left\|S_{\alpha}\left(l_{i+1}\right) \phi\right\|_{L^{2}(\Omega)}
\end{aligned}
$$

This leads to

$$
\begin{aligned}
& \frac{l_{i}-l_{i+1}}{4 C_{1} e^{C_{2} \sqrt{r_{i}}}}\left\|S_{\alpha}\left(l_{i}\right) \phi\right\|_{L^{2}(\Omega)} \\
& \quad-\frac{C_{1} e^{C_{2} \sqrt{r_{i}}}+1}{C_{1} e^{C_{2} \sqrt{r_{i}}}}\left(l_{i}-l_{i+1}\right) e^{-r_{i}^{\alpha}\left(\left(l_{i}-l_{i+1}\right) / 4\right)}\left\|S_{\alpha}\left(l_{i+1}\right) \phi\right\|_{L^{2}(\Omega)} \\
& \leq \int_{l_{i+1}}^{l_{i}} \chi_{E}(t)\left\|S_{\alpha}(t) \phi\right\|_{L^{2}(\omega)} d t .
\end{aligned}
$$

Summing (26) from $i_{0}$ to $\infty$, it follows that

$$
\begin{aligned}
& \frac{l_{i_{0}}-l_{i_{0}+1}}{4 C_{1} e^{C_{2} \sqrt{r_{0}}}}\left\|S_{\alpha}\left(l_{i_{0}}\right) \phi\right\|_{L^{2}(\Omega)}+\sum_{i=i_{0}}^{\infty} k_{i}\left\|S_{\alpha}\left(l_{i+1}\right) \phi\right\|_{L^{2}(\Omega)} \\
& \leq \int_{0}^{T} \chi_{E}(t)\left\|S_{\alpha}(t) \phi\right\|_{L^{2}(\omega)} d t,
\end{aligned}
$$

where

$$
\begin{array}{r}
k_{i}=\frac{l_{i+1}-l_{i+2}}{4 C_{1} e^{C_{2} \sqrt{r_{i+1}}}}-\frac{C_{1} e^{C_{2} \sqrt{r_{i}}}+1}{C_{1} e^{C_{2} \sqrt{r_{i}}}}\left(l_{i}-l_{i+1}\right) e^{-r_{i}^{\alpha}\left(\left(l_{i}-l_{i+1}\right) / 4\right)}, \\
i=i_{0}, i_{0}+1, i_{0}+2 \ldots
\end{array}
$$

Actually, by (17), we can derive that

$$
k_{i}>0, \quad \text { for any } i=i_{0}, i_{0}+1, i_{0}+2 \ldots
$$

This, together with (27), shows that

$$
\begin{aligned}
\left\|S_{\alpha}(T) \phi\right\|_{L^{2}(\Omega)} & \leq\left\|S_{\alpha}\left(l_{i_{0}}\right) \phi\right\|_{L^{2}(\Omega)} \\
& \leq \frac{4 C_{1} e^{C_{2} \sqrt{r_{0}}}}{l_{i_{0}}-l_{i_{0}+1}} \int_{E}\left\|S_{\alpha}(t) \phi\right\|_{L^{2}(\omega)} d t .
\end{aligned}
$$

Now, we are in the position to prove (7). The solution of (5) can be written as follows:

$$
\varphi(t)=S_{\alpha}(T-t) \varphi_{0}
$$

Along with (30), we can deduce that

$$
\|\varphi(x, 0)\|_{L^{2}(\Omega)} \leq \frac{4 C_{1} e^{C_{2} \sqrt{r_{i_{0}}}}}{l_{i_{0}}-l_{i_{0}+1}} \int_{F}\|\varphi(t)\|_{L^{2}(x, \omega)} d t .
$$

This completes the proof of the main result.

\section{Conflict of Interests}

The authors declare that there is no conflict of interests regarding the publication of this paper.

\section{Acknowledgments}

The authors would like to express their sincere thanks to the referees for their providing several important references and for their valuable suggestions. This work was partially supported by the National Research Foundation of South Africa under Grant CPR2010030300009918, the National Natural Science Foundation of China (U1204105, 61203293), and the Key Foundation of Henan Educational Committee (13A120524, 12B120006).

\section{References}

[1] R. Metzler and J. Klafter, "The restaurant at the end of the random walk: recent developments in the description of anomalous transport by fractional dynamics," Journal of Physics A: Mathematical and General, vol. 37, no. 31, pp. R161-R208, 2004.

[2] K. Sato, Levy Processes and infinitely Divisible Distributions, Cambridge Studies in Advanced Mathematics, Cambridge University Press, Cambridge, Mass, USA, 1999.

[3] J. Apraiz, L. Escauriaza, G. Wang, and C. Zhang, "Observability inequalitiesand measurable sets," Journal of the European Mathematical Society. In press.

[4] A. V. Fursikov and O. Yu. Imanuvilov, "Controllability of Evolution Equations," in Lecture Notes Series, vol. 34, Seoul National University, Seoul, South Korea, 1996.

[5] G. Wang, " $L^{\infty}$-null controllability for the heat equation and its consequences for the time optimal control problem," SIAM Journal on Control and Optimization, vol. 47, no. 4, pp. 17011720, 2008. 
[6] G. Wang and L. Wang, "The Carleman inequality and its application to periodic optimal control governed by semilinear parabolic differential equations," Journal of Optimization Theory and Applications, vol. 118, no. 2, pp. 429-461, 2003.

[7] C. Zhang, "An observability estimate for the heat equation from a product of two measurable sets," Journal of Mathematical Analysis and Applications, vol. 396, no. 1, pp. 7-12, 2012.

[8] J. Apraiz and L. Escauriaza, "Null-control and measurable sets," ESAIM: Control, Optimisation and Calculus of Variations, vol. 19, no. 1, pp. 239-254, 2013.

[9] S. Micu and E. Zuazua, "On the controllability of a fractional order parabolic equation," SIAM Journal on Control and Optimization, vol. 44, no. 6, pp. 1950-1972, 2006.

[10] L. Miller, "On the controllability of anomalous diffusions generated by the fractional Laplacian," Mathematics of Control, Signals, and Systems, vol. 18, no. 3, pp. 260-271, 2006.

[11] J. L. Lions, "Exact controllability, stabilization and perturbations for distributed systems," SIAM Review, vol. 30, no. 1, pp. 1-68, 1988.

[12] A. Pazy, Semigroups of Linear OperaTors and Applications To Partial Di Erential Equations, Springer, New York, NY, USA, 1983.

[13] K. D. Phung and G. Wang, "An observability estimate for the parabolic equations from a measurable set in time and its applications," Journal of the European Mathematical Society, vol. 15, pp. 681-703, 2013. 


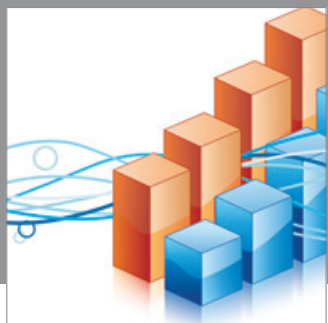

Advances in

Operations Research

mansans

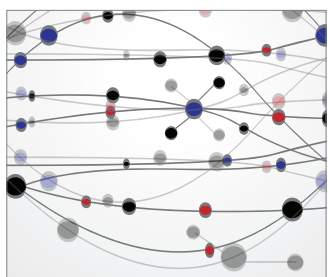

The Scientific World Journal
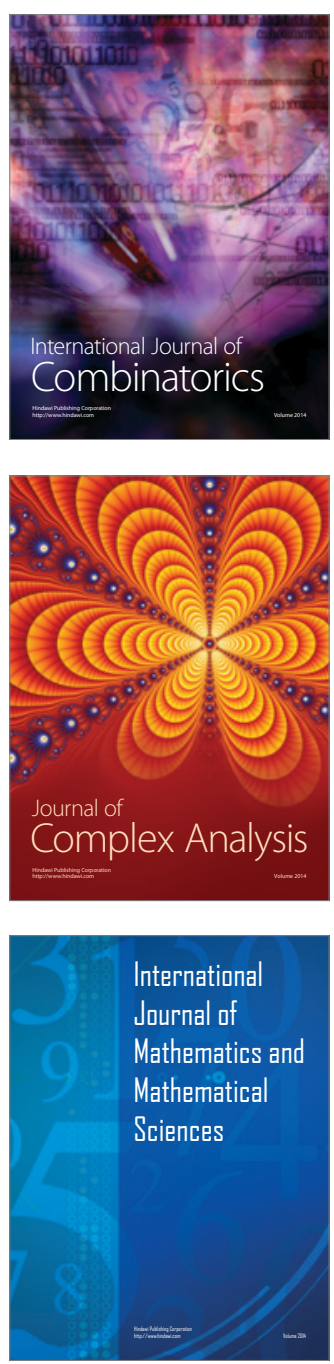
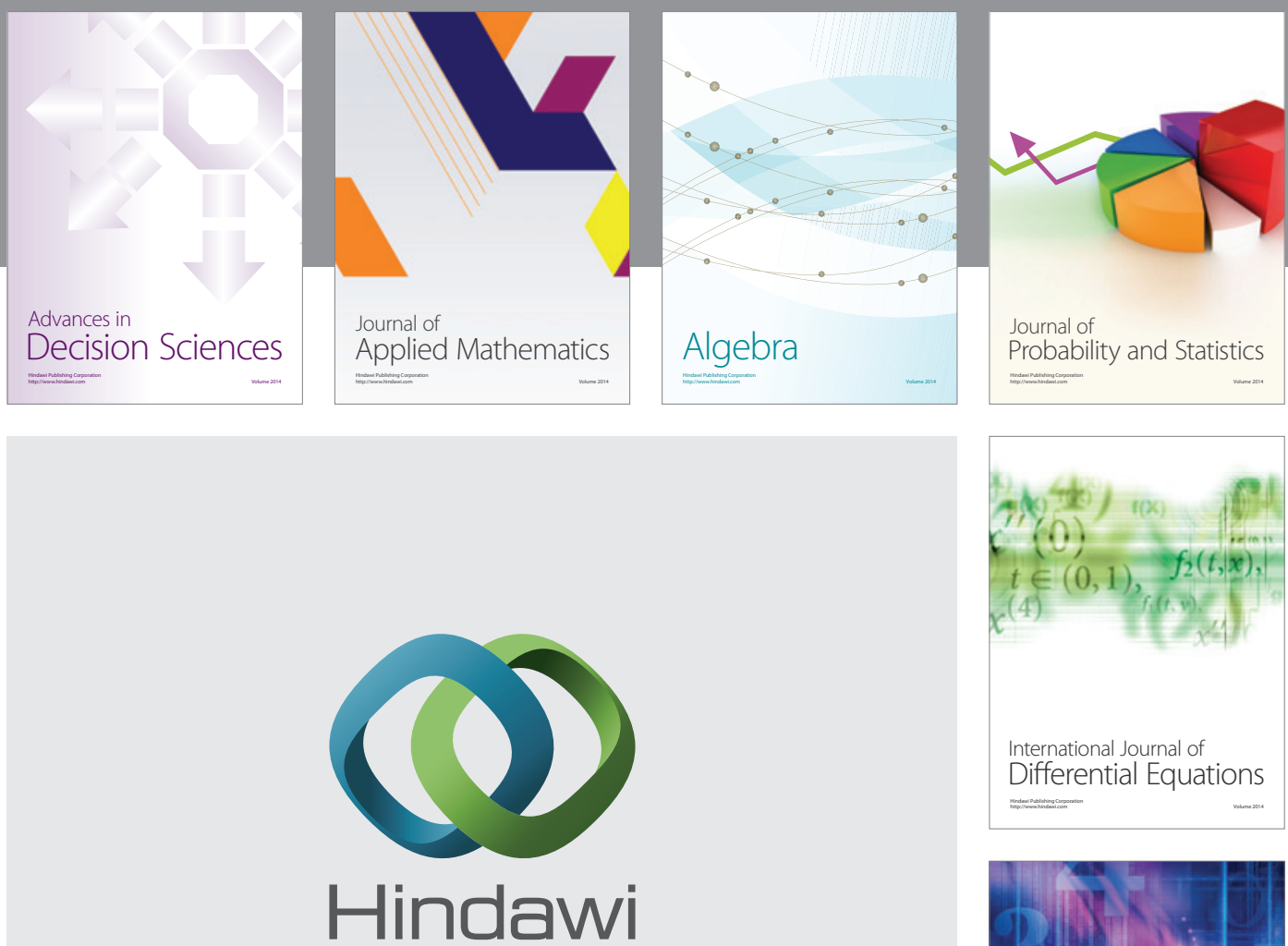

Submit your manuscripts at http://www.hindawi.com
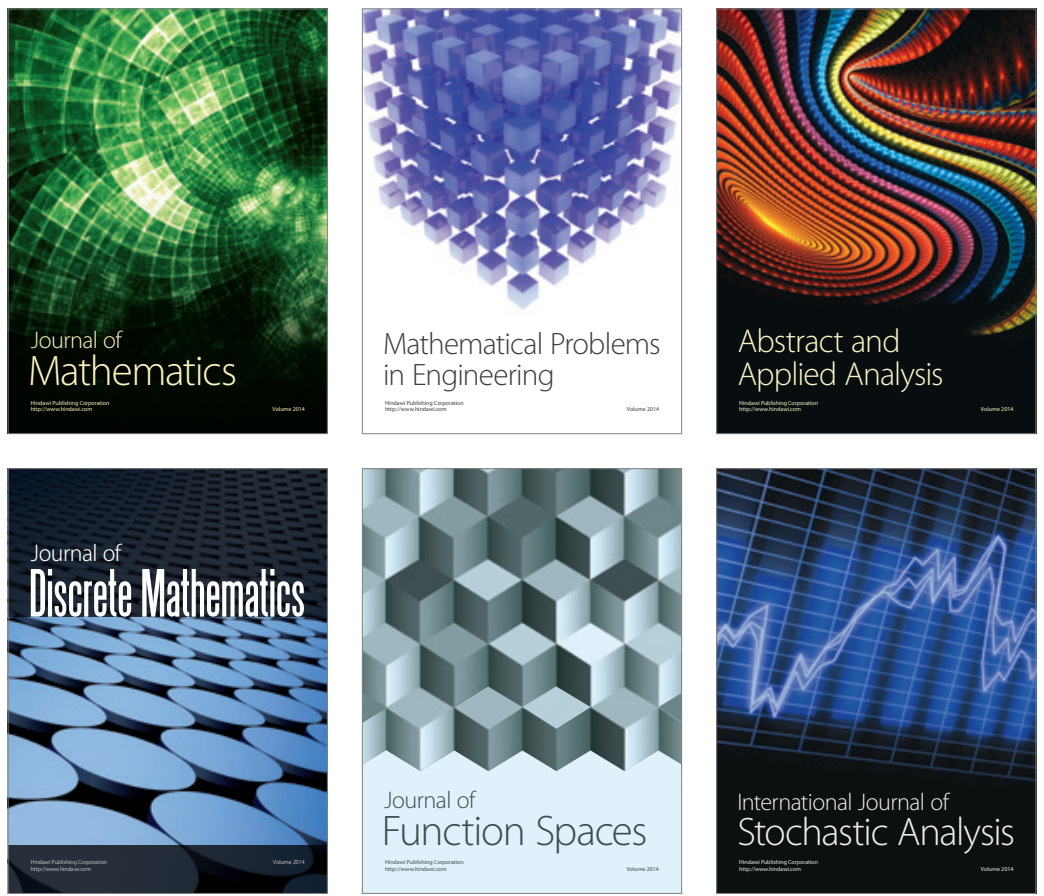

Journal of

Function Spaces

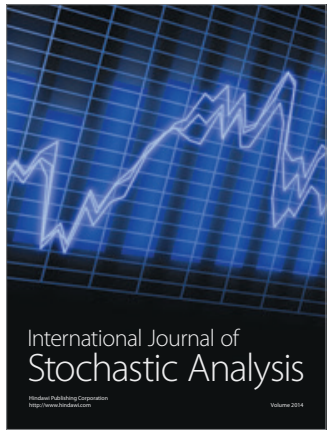

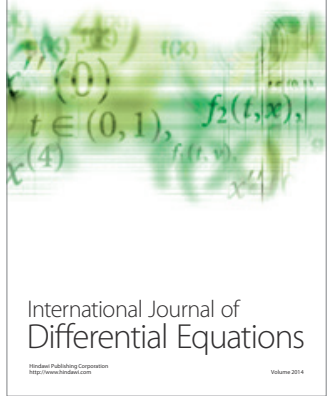
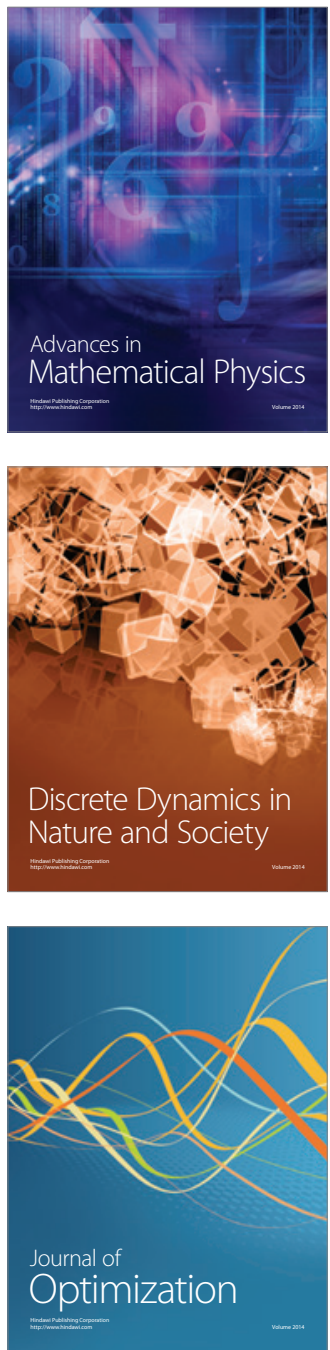